\title{
Mortality and Major Morbidities of Very Low Birth Weight Infants at a Teaching Hospital in Saudi Arabia: A Comparison of Two Periods
}

\author{
Khalid Altirkawi Nasser Alyousef Badr Sobaih Adnan J. Alhadid \\ Lana Shaiba Rozina Banoo Amull Fariss \\ Department of Pediatrics, College of Medicine/King Saud University, Riyadh, Saudi Arabia
}

\section{Keywords}

Outcomes · Mortality · Preterm infant · Very low birth weight infant

\begin{abstract}
Objectives: To assess the status of care provided in the neonatal intensive care unit at our institution and to track the changes in rates of mortality and major morbidities of very low birth weight infants, delivered over two periods. Materials and Methods: We analyzed retrospectively the records of preterm infants, with birth weights $<1,500 \mathrm{~g}$ and gestational age $<33$ weeks. Infants studied were born over two periods; the first extends from January 1999 to December 2007 and the second from January 2011 to December 2018. We compared the rates of survival to discharge and major morbidities of the infants in these two periods and, as a corollary, to the rates published in 2007 by the National Institute of Child Health and Human Development (NICHD) neonatal research network. Results: Five hundred and twelve infants were included in the second period of this study. The survival-todischarge rate was $84 \%$, and it varied among infants based on their gestational ages. Generally, morbidities reported in the second period were less than their counterparts in the first one and comparable to NICHD ones. The most common morbidities were respiratory distress syndrome, $88.5 \%$; reti-
\end{abstract}

karger@karger.com www.karger.com/dmj

Karger $\stackrel{\text { ' }}{5}$
(C) 2021 The Author(s)

Published by S. Karger AG, Basel

This is an Open Access article licensed under the Creative Common Attribution-NonCommercial-4.0 International License (CC BY-NC) (http://www.karger.com/Services/OpenAccessLicense), applicable to the online version of the article only. Usage and distribution for commercial purposes requires written permission. nopathy of prematurity, 28.3\%; patent ductus arteriosus (PDA), 27.9\%; and sepsis/meningitis, 25.6\%. Conclusion: Most of the assessed morbidity rates have decreased over time, except for periventricular leukomalacia which increased. The survival-to-discharge rates have not improved as expected; this trend seems concerning and warrants further investigation.

(c) 2021 The Author(s)

Published by S. Karger AG, Basel

\section{Introduction}

Improvements of obstetric and perinatal care, including the use of antenatal corticosteroids and surfactant replacement therapy, paralleled an increase in survival of very low birth weight (VLBW) infants [1]. The emergence of new modes of respiratory support and establishment of quality and patients' safety culture might have contributed to this improvement too [2]. However, despite these advances, rates of major morbidities remain unduly high, leading to higher chances of adverse neurodevelopmental outcomes, as their occurrence seems to be directly related to the rates of neonatal morbidities [3]. Assessing the impact of prematurity on mortality rates and the short- and long-term outcomes is important for providing families with relevant and accurate counseling, both antenatally and postnatally, 
to improve the decision-making process and, hopefully, to advance the survival without impairments [4].

Furthermore, continued monitoring of mortality and morbidity rates enables institutions to benchmark their performance and to discover areas that need further efforts to improve [5]. Unfortunately, despite the plethora of studies in this area, there is a paucity of such studies reporting on the local burden of prematurity and its short- and long-term outcomes $[6,7]$.

The primary objective of this study is to compare the rates of major morbidities and survival to discharge of VLBW infants, admitted to the neonatal intensive care unit (NICU) at King Saud University Medical City (KSUMC) over two periods; the first extends from January 1999 to December 2007 and the second extends from January 2011 to December 2018. Previously, the data collected in the first period were benchmarked to rates published in 2007 by the National Institute of Child Health and Human Development (NICHD) neonatal research network. The results of that comparison were included in a study published in 2014 [8].

\section{Materials and Methods}

We conducted a retrospective analysis of prospectively collected data over an 8-year period, spanning the years 2011 through 2018. In compliance with the international ethical standards and local regulations governing research on human subjects, all personal identifiers of infants included in this study were removed before analysis was performed; collected data were stored in one computer set within the premises of KSUMC and kept accessible only to the authorized investigator. We included in this analysis all infants admitted to NICU at KSUMC with a birth weight $<1,500 \mathrm{~g}$ and gestational age $<33$ weeks. We excluded all infants with congenital anomalies and syndromes that were considered lethal. The data collected included maternal characteristics (age and parity), pregnancy and labor issues (antenatal exposure to steroids, antenatal administration of antibiotics, and rupture of fetal membranes), perinatal issues (mode of delivery, the need for resuscitation and intubation, Apgar's scores, and infant's temperature upon admission), neonatal demographic features (infant's sex, gestational age, and birth weight), postnatal management (surfactant replacement therapy and the length of hospital stay), and finally infants' survival to discharge grouped according to their gestational ages.

The case definitions of major morbidities assessed were kept similar to their counterparts in the first period (1999-2007). Thus, respiratory distress syndrome (RDS) was diagnosed if the infant exhibited clinical signs of respiratory distress, needed supplemental oxygen, and presented radiological changes on the chest radiograph consistent with RDS. The diagnosis of pneumothorax was based on the presence of intrapleural air collection observed on the chest radiograph, or obtained by needle aspiration of the pleural space. Bronchopulmonary dysplasia (BPD) was defined as the

Short-Term Outcomes of VLBW Infants need for oxygen supplementation at the corrected gestational age of 36 weeks [9].

Patent ductus arteriosus (PDA) was diagnosed via echocardiography with an evidence of left-to-right shunting. The diagnosis of intraventricular hemorrhage (IVH) was made via a head ultrasonographic scanning performed during the first 4 weeks of life and graded according to Papile's classification [10]. When multiple ultrasonographic scans were performed, the worst grade reported was considered for this analysis. Periventricular leukomalacia (PVL) was diagnosed upon detecting the characteristic periventricular echogenicity on head ultrasonographic scanning performed at any time during the hospital stay.

Sepsis diagnosis was based on having the growth of a pathogenic organism on blood culture obtained in the context of clinical finding consistent with sepsis. The early-onset sepsis was defined as sepsis diagnosed in the first $72 \mathrm{~h}$ of life. Diagnosis of meningitis was based on the presence of a pathogen growth on a cerebrospinal fluid culture, obtained in the presence of clinical findings suggestive of sepsis/meningitis.

Necrotizing enterocolitis (NEC) was diagnosed based on clinical manifestations, such as abdominal distension and intolerance of feeds or bloody stools, in addition to an abdominal radiograph showing pneumatosis intestinalis, pneumoperitoneum, or gas in the biliary tree. Thus, cases of presumed NEC without radiological evidence were excluded. Bell's classification was used for severity assignment [11].

Diagnosis of retinopathy of prematurity (ROP) was based on retinal examination performed at the age set by our NICU protocol and was graded according to the International Classification of ROP [12]. The mortality rate of interest was death prior to hospital discharge. The total rates of mortality and survival to discharge were further stratified based on infants' gestational ages.

\section{Statistical Analysis}

We assessed the mortality rates in association with different gestational ages, but morbidities were not. All variables in the second period were compared to their counterparts in the first one and, as a corollary, to the rates published by the NICHD neonatal research network. The statistical analysis was performed using IBM SPSS statistics, version 22.0 (IBM Inc., Armonk, NY, USA). Results of continuous variables were presented as means and standard deviations, and those of categorical variables as frequencies and percentages. Tests of significance ( $t$ test, Fisher's exact test, and $\chi^{2}$ test) were used as appropriate and set at $p \leq 0.05$.

\section{Results}

Five hundred and twelve infants were eligible for inclusion in this study (Table 1). Of them, 53\% were male infants. Although mean birth weight of these infants was 
Table 1. Demographics and perinatal characteristics presented as means (SD)

\begin{tabular}{lclc}
\hline & First period & Second period & $p$ value \\
\hline Birth weight (95\% CI), g & $993(287)$ & $1,038(276)$ & 0.011 \\
Gestational age, weeks & $27.7(2.41)$ & $27.9(2.42)$ & 0.195 \\
Maternal age, years & $28.9(6.1)$ & $29.1(5.7)$ & 0.595 \\
Parity & $1(0.1)$ & $1.02(0.17)$ & 0.03 \\
Apgar's scores & $5(2)$ & $5.7(1.8)$ & $<0.0001$ \\
$\quad 1$ min & $7.4(1.5)$ & $7.7(1.4)$ & 0.0012 \\
$\quad 5$ min & $35.7(1)$ & $36.2(0.4)$ & $<0.0001$ \\
Temperature upon admission, ${ }^{\circ} \mathrm{C}$ & $71(53.2)$ & $73.6(90.6)$ & 0.588 \\
Length of hospital stay, days & $2,357.4(739.5)$ & $2,507(1,066)$ & 0.0116 \\
Weight on discharge, $\mathrm{g}$ & & & \\
\hline
\end{tabular}

Table 2. Survival-to-discharge rates based on gestational age

\begin{tabular}{|c|c|c|c|c|c|}
\hline \multirow[t]{2}{*}{ Gestational age } & \multicolumn{2}{|c|}{ First period } & \multicolumn{2}{|c|}{ Second period } & \multirow[t]{2}{*}{$p$ value } \\
\hline & $\begin{array}{l}\text { survived/ } \\
\text { total, } n / N\end{array}$ & $\begin{array}{l}\text { survival } \\
\text { rate, } \%\end{array}$ & $\begin{array}{l}\text { survived/ } \\
\text { total, } n / N\end{array}$ & $\begin{array}{l}\text { survival rate, } \\
\%\end{array}$ & \\
\hline 22 weeks & $0 / 1$ & 0 & $0 / 2$ & 0 & NS \\
\hline 23 weeks & $11 / 18$ & 61 & $7 / 17$ & 41.2 & 0.248 \\
\hline 24 weeks & $26 / 40$ & 65 & $15 / 37$ & 40.5 & 0.032 \\
\hline 25 weeks & $28 / 33$ & 84.8 & $27 / 35$ & 77.1 & 0.423 \\
\hline 26 weeks & $63 / 70$ & 90 & $44 / 60$ & 73.3 & 0.013 \\
\hline 27 weeks & $44 / 49$ & 90 & $46 / 49$ & 93.9 & 0.480 \\
\hline 28 weeks & $58 / 62$ & 93.6 & $85 / 94$ & 90.4 & 0.480 \\
\hline 29 weeks & $54 / 59$ & 91.5 & $60 / 67$ & 89.6 & 0.717 \\
\hline 30 weeks & $77 / 78$ & 98.7 & $76 / 80$ & 95 & 0.186 \\
\hline 31 weeks & $39 / 43$ & 91 & $29 / 30$ & 96.7 & 0.339 \\
\hline 32 weeks & $15 / 15$ & 100 & $41 / 41$ & 100 & NS \\
\hline All ages & $415 / 468$ & 88.7 & $430 / 512$ & 84 & 0.029 \\
\hline
\end{tabular}

significantly higher than their counterparts in the first period $(1,038 \mathrm{~g}$ [95\% CI: 1,013-1,062] vs. $993 \mathrm{~g}$ [95\% CI: $968-1,018], p=0.011)$, their mean gestational age was not ( 27.9 vs. 27.7 weeks, $p=0.195$ ). Apgar's scores of infants born in the second period were significantly higher (1st min: 5.7 [95\% CI: 5.54-5.86] vs. 5 [95\% CI: 4.8-5.18], $p<$ 0.0001 ; 5th min: 7.7 [95\% CI: 7.57-7.83] vs. 7.4 [95\% CI: 7.27-7.53], $p=0.0012)$. And, the same is true for the infants' temperature upon admission to NICU, $36.2^{\circ} \mathrm{C}$ (95\% CI: $36.16-36.24)$ versus $35.7^{\circ} \mathrm{C}$ (95\% CI: $35.6-$ 35.79), $p<0.0001$.

Four hundred and thirty infants survived to discharge: a survival rate of $84 \%$, a drop from its level in the first period of $88.7 \%, p=0.029$. The 2 infants born at 22 weeks of gestation and included in this study died prior to discharge (Table 2). However, survival rates of infants born at 23 and 24 weeks of gestation were $41.2 \%$ and $40.5 \%$, respectively. Of note, we offer active resuscitation for all extremely low birth weight infants born at our institution, provided the infant was born at/after 22 weeks of gestation mark or of a birth weight of $\geq 500 \mathrm{~g}$.

As Table 3 shows, there were no significant differences between the rates of intrapartum antibiotic prophylaxis administered to mothers in these two periods, despite the significant rise in the rates of premature $(\geq 24 \mathrm{~h})$ rupture of membranes $(18.9 \%$ vs. $3.5 \%, p<0.0001)$ and cesarean section deliveries ( $58.6 \%$ vs. $47.8 \%, p=0.0007)$ in the second period. The rate of antenatal corticosteroid use in deliveries complicated with prematurity continued to increase, though at a slower pace $(81.5 \%$ vs. $77.5 \%, p=0.12)$.

Table 4 summarizes the incidence rates of the major morbidities in both periods. We observed significant drops in the rates of RDS diagnosis, surfactant replacement therapy, and postnatal corticosteroids therapy. The rate of $\mathrm{BPD}$, however, has not changed significantly (23.6\% vs. $27.4 \%, p=0.172)$, and the same is true for the composite rate of "death or BPD" (39.6\% vs. $38.6 \%, p=$ $0.70)$. 
Table 3. Comparison of pregnancy, labor, and delivery variables between the second period (2011-2018) and the first period (1999-2007) and the NICHD 2007 cohort

\begin{tabular}{|c|c|c|c|c|}
\hline & $\begin{array}{l}\text { NICHD } 2007 \\
\text { cohort* }\end{array}$ & $\begin{array}{l}\text { KSUMC } \\
\text { first period* }\end{array}$ & $\begin{array}{l}\text { KSUMC } \\
\text { second period }\end{array}$ & $p$ value $\dagger$ \\
\hline$N$ & 18,150 & 512 & 468 & \\
\hline Survival rate, $\%$ & 85 & 88.7 & 84 & 0.029 \\
\hline \multicolumn{5}{|l|}{ Delivery room resuscitation, \% } \\
\hline Endotracheal intubation & 53 & 75.4 & 56.8 & $<0.0001$ \\
\hline Use of resuscitation drugs & 5 & 3.4 & 0.4 & 0.0005 \\
\hline \multicolumn{5}{|l|}{ Mode of delivery, $\%$} \\
\hline SVD & 42 & 52.2 & 41.4 & 0.0007 \\
\hline Caesarean section & 58 & 47.8 & 58.6 & 0.0007 \\
\hline \multicolumn{5}{|l|}{ Others, $\%$} \\
\hline Antenatal corticosteroids & 79 & 77.5 & 81.5 & 0.120 \\
\hline Use of IAP & 70 & 33.8 & 33.4 & 0.894 \\
\hline PROM & 24 & 3.5 & 18.9 & $<0.0001$ \\
\hline Multiple births & 26 & 29.6 & 29.3 & 0.918 \\
\hline SGA & 21 & 12.5 & 11.5 & 0.630 \\
\hline
\end{tabular}

SVD, spontaneous vaginal delivery; IAP, intrapartum antibiotic prophylaxis; PROM, premature rupture of membranes; SGA, small for gestational age. ${ }^{*}$ Data are extracted with permission from Sobaih et al. [8]. †p value is for direct comparisons made solely between the first and the second period groups.

Although the diagnosis of PDA has not changed significantly between the two periods $(27.9 \%$ vs. $31 \%, p=$ 0.287 ), the patients of the second period were managed in a more conservative manner and received less medical $(16.6 \%$ vs. $48.3 \%, p=0.0001)$ and surgical (2.2\% vs. $12.4 \%$, $p=0.0001$ ) interventions. Collectively, IVH of all grades was detected in $13.2 \%$ of these infants, a nonsignificant decrease from $13.9 \%$ reported in the first period, so was the rate of severe cases (grades III and IV), which dropped from $7.8 \%$ to $7 \%$. On the contrary, infants of the second period were more likely to have the diagnosis of PVL as compared to their counterparts of the first period $(2.5 \%$ vs. $1.3 \%, p=0.172$ ). The incidence of severe ROP in the second period was $18 \%$; however, the diagnosis of ROP of any stage dropped in the second period from $34.5 \%$ to $28.3 \%(p=0.036)$. Finally, we observed a significant drop in the rates of both early-onset sepsis $(1.6 \%$ vs. $11 \%, p<$ $0.0001)$ and late-onset sepsis ( $24 \%$ vs. $34.5 \%, p<0.0001)$, but no such changes were noted in the incidence of NEC, which was diagnosed in $16 \%$ of these infants.

\section{Discussion}

The decreased incidence rates of most morbidities and short-term adverse outcomes in the second period are a testimony to the efficacy of several changes of care we implemented over the past few years. The incidence of RDS is a good example in this regard; although it is significantly higher than NICHD published figures [1], it continued to decrease, with increased use of antenatal corticosteroids in the context of deliveries complicated with prematurity. This decrease in RDS occurrence might have contributed to the mild decrease in the incidence rate of BPD as well.

Apparently, the pathogenesis of BPD is multifactorial, but adopting less-invasive approaches in managing respiratory failure in these infants, as manifested by decreased rate of endotracheal intubation $(56.8 \%$ vs. $75.4 \%, p<$ 0.0001 ), is expected to mitigate its incidence. Notably, our management protocols changed over time to adopt noninvasive modes of ventilation and earlier use of continuous positive airway pressure in the delivery room and emphasized the adherence to evidence-based neonatal resuscitation practices. Interestingly, despite the less frequent use of surfactant replacement therapy and postnatal corticosteroids during the second period of this study, the incidence rate of BPD was not adversely affected, and the rate of "BPD or death" remained almost unchanged. One has to keep in mind, however, that infants in the second period have higher birth weights, which may have contributed to this favorable outcome.

The diagnosis of ROP of all stages decreased significantly in the second period. This change may be ascribed, 
Table 4. Short-term outcomes of infants born in the second period (2011-2018) in comparison with those born in the first period (1999-2007) and the NICHD 2007 cohort

\begin{tabular}{|c|c|c|c|c|}
\hline & $\begin{array}{l}\text { NICHD cohort } \\
(2007)^{*}\end{array}$ & $\begin{array}{l}\text { KSUMC } \\
\text { 1st period* }\end{array}$ & $\begin{array}{l}\text { KSUMC } \\
\text { 2nd period }\end{array}$ & $p$ value $\dagger$ \\
\hline$N$ & 18,150 & 468 & 512 & \\
\hline RDS, \% & 44 & 95.2 & 88.5 & $<0.0001$ \\
\hline Surfactant therapy, \% & 58 & 80 & 66.6 & $<0.0001$ \\
\hline Postnatal corticosteroids, $\%$ & 17 & 27.9 & 8 & $<0.0001$ \\
\hline Pneumothorax, \% & 5 & 5 & 6.5 & 0.315 \\
\hline $\mathrm{BPD}, \%$ & 22 & 27.4 & 23.6 & 0.172 \\
\hline BPD or death, $\%$ & 37 & 38.6 & 39.6 & 0.70 \\
\hline \multicolumn{5}{|l|}{ PDA, \% } \\
\hline Incidence & 29 & 31 & 27.9 & 0.287 \\
\hline Medical treatment & 79 & 48.3 & 16.6 & $<0.0001$ \\
\hline Surgical intervention & 19 & 12.4 & 2.2 & $<0.0001$ \\
\hline Cranial sonogram done, $\%$ & 93 & 90 & 100 & $<0.0001$ \\
\hline \multicolumn{5}{|l|}{ IVH, \% } \\
\hline Grade I & 11 & 2.7 & 3.7 & 0.376 \\
\hline Grade II & 4 & 3.4 & 2.5 & 0.404 \\
\hline Grade III & 7 & 4.4 & 3.5 & 0.469 \\
\hline Grade IV & 5 & 3.4 & 3.5 & 0.931 \\
\hline PVL, \% & 3 & 1.3 & 2.5 & 0.172 \\
\hline \multicolumn{5}{|l|}{ Sepsis, \% } \\
\hline EOS & 2 & 11 & 1.6 & $<0.0001$ \\
\hline LOS & 22 & 37.2 & 24 & $<0.0001$ \\
\hline NEC, $\%$ & 7 & 15.6 & 16 & 0.863 \\
\hline ROP (all stages), \% & 59 & 34.5 & 28.3 & 0.036 \\
\hline
\end{tabular}

RDS, respiratory distress syndrome; BPD, bronchopulmonary dysplasia; PDA, patent ductus arteriosus; IVH, intraventricular hemorrhage; PVL, periventricular leukomalacia; EOS, early-onset sepsis; LOS, late-onset sepsis; NEC, necrotizing enterocolitis; ROP, retinopathy of prematurity. *Data are extracted with permission from Sobaih et al. [8]. $\dagger p$ value is for direct comparisons made solely between the first and the second period groups.

at least partially, to the careful use of supplemental oxygen in resuscitation and management of these failing infants. Unfortunately, as the number of severe ROP cases in the first period was lacking, we were unable to make a direct comparison of this variable between the two groups. Nonetheless, the rate of ROP cases that needed ablation therapy in the second period (18\%) was comparable to what is reported in the recent literature $[13,14]$, a reflection of the robust screening program implemented in our unit.

The incidence rate of IVH, which is already lower than NICHD reported rates, has not changed significantly between the two periods, but a trend towards milder grades is noticeable. In contrast to the international trend, our practice to use indomethacin prophylaxis in extremely low birth weight infants has not changed after the publication of the TIPP trial [15]. This practice, we speculate, may have played a role in maintaining such low rates of IVH in this cohort of infants. On the other hand, the in- cidence rate of PVL in the second period increased insignificantly $(2.5 \%$ vs. $1.3 \%, p=0.172)$. Although it is still lower than what is reported by the NICHD (3\%), it sounds alarming and deserves further investigations.

The rates of medical treatment of PDA dropped significantly ( $16.6 \%$ vs. $48.3 \%, p<0.0001)$, so does the rate of its surgical interventions ( $2.2 \%$ vs. $12.4 \%, p<0.0001)$. Nonetheless, the incidence rate of PDA shows a less impressive decrease. We hypothesize that adopting newer guidelines that favor a more targeted therapeutic approach has played a role in these changes. This observation lends support to more tolerant approaches towards PDA management.

The observed changes in the rates of sepsis/meningitis in our unit are very encouraging. Arguably, the significant drop in the incidence rate of late-onset sepsis $(24 \%$ vs., $37.2 \%, p<0.0001$ ) could be credited to the rigorous infection control measures and the ongoing quality improvement project aiming to reduce the neonatal sepsis; 
however, the drop in the incidence of early-onset sepsis $(1.6 \%$ vs. $11 \%, p<0.0001)$ seems more likely to reflect the improved levels of prenatal care and obstetrical practices in our institution and the community at large.

While some fluctuation in survival rates is expected, as reported by Cheong et al. [16], the survival-to-discharge rates in the second period were less encouraging, especially among infants born close to the border of viability; they dropped significantly ( $84 \%$ vs. $88.7 \%, p=0.029$ ). This observation may be attributed to the many changes that took place in our unit recently, such as the increased capacity from 23 to 44 beds and the policy changes that led to a net increase of sicker infants admitted/readmitted to NICU through the emergency room, or transferred from peripheral hospitals. Furthermore, the establishment of the fetomaternal unit at KSUMC added a significant number of high-risk pregnancies to the load of cases that were managed and eventually delivered in our hospital. These changes in the unit structure and policy entailed that our patient mix included a higher percentage of sicker infants and, possibly, shifted the rates of mortality to a higher level.

Other factors that might have contributed to the increased mortality in the second period include the higher proportions of high-multiple births, especially triplets, as a result of more in vitro fertilization cases and those of "unbooked" pregnancies (32\% of all deliveries), which increase the chances of perinatal and neonatal complications. Lastly, the increased rates of cesarean section deliveries $(58.6 \%$ vs. $47.8 \%, p=0.0007)$, especially the emergency ones, might have contributed, indirectly, to the observed increase in the mortality rates too.

Although our study reports on one of the largest cohorts of VLBW infants in the country, the retrospective nature of this study, in addition to the heterogeneity in the resources and adopted neonatal practices, hampers the ability to generalize these findings to a national level. Furthermore, data pertaining to some of the important morbidity variables (e.g., lethal congenital conditions) were not assessed, whereas others (e.g., ROP) were inadequately collected or being truncated to match their counterparts in the first period (e.g., BPD). Therefore, detailed, more nuanced, and standardized data, collected on mortality and major morbidities of VLBW infants in Saudi Arabia, are still needed; such an endeavor is already in consideration as we prepare for the next iteration of this report.

\section{Conclusions}

Improvements in the neonatal and antenatal care provided to the VLBW infants in our institution led to significant drops in the rates of major morbidities affecting them; nonetheless, their survival-to-discharge rates have not followed suit. Many changes in the NICU policies and structure of care delivered seem to play a role in the increased mortality in the later period.

\section{Statement of Ethics}

The Institutional Review Board (IRB) at King Saud University Medical City (KSUMC), Reference No. 20/0076/IRB, September 20, 2020, approved this study protocol. The study conformed to the tenets of the Declaration of Helsinki. As the present study was carried out retrospectively, the need to obtain written informed consent from the patients other than the general patient's consent to treatment was waived by the IRB. All personal identifiers of the patients were removed, and the data were analyzed anonymously.

\section{Conflict of Interest Statement}

The authors have no conflicts of interest to declare.

\section{Funding Sources}

The authors did not receive any funding.

\section{Author Contributions}

K.A., the principal investigator, contributed to the study design, data analysis, writing the early and final versions of the manuscript, and journal correspondence. N.A. performed data collection, statistical analysis, and contributed to writing of the manuscript. B.S. provided the data set and contributed to the methods design. A.J.A. and L.S. both contributed to the critical reading of data and manuscript early versions. R.B. and A.F. contributed mainly in data collection and early stages of analysis.

\section{References}

1 Fanaroff AA, Stoll BJ, Wright LL, Carlo WA, Ehrenkranz RA, Stark AR, et al. NICHD neonatal research network. Trends in morbidity and mortality for very low birthweight infants. Am J Obstet Gynecol. 2007;196:147.e1-8.
2 Abolfotouh MA, Al Saif S, Altwaijri WA, Al Rowaily MA. Prospective study of early and late outcomes of extremely low birthweight in Central Saudi Arabia. BMC Pediatr. 2018;18: 280.
3 Stoll BJ, Hansen NI, Bell EF, Shankaran S, Laptook AR, Walsh MC, et al. Neonatal outcomes of extremely preterm infants from the NICHD neonatal research network. Pediatrics. 2010;126(3):443-56. 
4 Patel RM. Short- and long-term outcomes for extremely preterm infants. Am J Perinatol. 2016 Feb;33(3):318-28.

5 Mesleh RA, Kurdi AM, Sabagh TO, Algwiser AA. Changing trends in perinatal deaths at the armed forces hospital, Riyadh, Saudi Arabia. J Obstet Gynaecol. 2001 Jan;21(1):49-55.

6 Al-Qurashi FO, Yousef AA, Awary BH. Epidemiological aspects of prematurity in the Eastern region of Saudi Arabia. Saudi Med J. 2016;37(4):414-9.

7 Al Hazzani F, Al-Alaiyan S, Hassanein J, Khadawardi E. Short-term outcome of very lowbirth-weight infants in a tertiary care hospital in Saudi Arabia. Ann Saudi Med. 2011;31(6): 581-5.

8 Sobaih B, Hadid A, Fariss A, Banoo R, Alkharfi T, Alfaleh K. Mortality and shortterm outcome of very low birth weight (VLBW) infants at a tertiary care center in Saudi Arabia: 9 years' data. Kuwait Med J. 2014;46(3):233-6.
9 Bancalari E, Calure N, Sosenko IR. Bronchopulmonary dysplasia: changes in pathogenesis, epidemiology and definition. Semin Neonatol. 2003;8:63-71.

10 Papile LA, Burstein J, Burstein R, Koffler H. Incidence and evolution of subependymal and hemorrhage: a study of infants with birth weights less than 1,500 gm. J Pediatr. 1978;92: 529-34.

11 Bell MJ, Ternberg JL, Feigin RD, Keating JP, Marshall R, Barton L, et al. Neonatal necrotizing enterocolitis. Therapeutic decisions based upon clinical staging. Ann Surg. 1978 Jan; 187(1):1-7.

12 International Committee for the Classification of Retinopathy of Prematurity. The international classification of retinopathy of prematurity revisited. Arch Ophthalmol. 2005; 123(7):991-9.
13 Freitas AM, Mörschbächer R, Thorell MR, Rhoden EL. Incidence and risk factors for retinopathy of prematurity: a retrospective cohort study. Int J Retina Vitreous. 2018;4(20): 20.

14 Celebi AR, Petricli IS, Hekimoglu E, Demirel $\mathrm{N}$, Bas AY. The incidence and risk factors of severe retinopathy of prematurity in extremely low birth weight infants in Turkey. Med Sci Monit. 2014;20:1647-53.

15 Clyman RI, Saha S, Jobe A, Oh W. Indomethacin prophylaxis for preterm infants: the impact of 2 multicentered randomized controlled trials on clinical practice. J Pediatr. 2007;150(1):46-50.e2.

16 Cheong JLY, Anderson PJ, Burnett AC, Roberts G, Davis N, Hickey L, et al. Changing neurodevelopment at 8 years in children born extremely preterm since the 1990s. Pediatrics. 2017;139(6):e20164086. 